\title{
芳甲基叠氮化合物与烯烃及其衍生物反应研究进展
}

\author{
李秀英 ${ }^{\dagger}, a$ 李亚军,$b$ 韦贤生 ${ }^{a}$ 罗金荣 ${ }^{a}$ 黄国保*,a 谭明雄 ${ }^{*, a}$ \\ $\left({ }^{a}\right.$ 广西农产资源化学与生物技术重点实验室 玉林师范学院化学与食品科学学院 玉林 537000) \\ ( ${ }^{b}$ 湖南环境生物职业技术学院医药技术学院 衡阳 421005)
}

\begin{abstract}
摘要 芳甲基叠氮化合物 $\left(\mathrm{ArCH}_{2} \mathrm{~N}_{3}\right)$ 可以为有机反应提供氮源，是一种性质稳定、合成简单的试剂，在有机合成化学领 域被广泛应用. 综述了最近五年来芳甲基叠氮化合物与烯烃在有机合成反应中的最新研究进展. 分别叙述了芳甲基叠 氮化合物在有机反应中与烯烃及其衍生物的底物适应范围和反应机理, 为今后芳甲基叠氮化合物与烯烃类化合物的反 应在有机合成中的应用提供参考.
\end{abstract}

关键词＼cjkstart芳甲基叠氮化合物; 烯烃; 反应机理

\section{Recent Progress on Reactions of Arylmethyl Azides with Alkenes}

\author{
Li, Xiuying ${ }^{\dagger, a}$ \\ Li, Yajun ${ }^{\dagger, b}$ \\ Huang, Guobao*,a \\ Wei, Xiansheng ${ }^{a}$ \\ Luo, Jinrong $^{a}$ \\ Tan, Minxiong ${ }^{*, a}$ \\ ( ${ }^{a}$ Guangxi Key Laboratory of Agricultural Resources Chemistry and Biotechnology, College of Chemistry and \\ Food Science of Yulin Normal University, Yulin 537000) \\ ( ${ }^{b}$ College of Medicine \& Technology, Hunan Polytechnic of Environment and Biology, Hengyang 421005)
}

\begin{abstract}
Arylmethyl azides $\left(\mathrm{ArCH}_{2} \mathrm{~N}_{3}\right)$ as one of the significant nitrogen sources with stable properties, simple synthesis, have been widely used in a wide range of organic synthesis reactions. The recent progress $(2014 \sim 2018)$ on reactions of arylmethyl azides with alkenes is summarized. In addition, the organic reactions of arylmethyl azides with types of alkenes are described respectively, with their scope of substrates and reaction mechanism. It is hoped that this review can be referred to the future application in organic synthesis of arylmethyl azides with alkenes.

Keywords arylmethyl azides; alkenes; reaction mechanism
\end{abstract}

自 1864 年科学家们首次发现有机叠氮化合物以 来, 因其独特的化学性质被广泛应用于有机合成化学、 药物化学以及材料科学等研究领域 ${ }^{[1]}$. 叠氮化合物由于 具有 1,3-偶极作用, 在不同反应条件下可以转化成胺、 酰胺、氮烯、亚胺、重氮化合物以及含氮杂环化合物 等 $^{[2]}$, 可以为有机反应提供 $\mathrm{N}$ 源. 芳甲基叠氮化合物作 为一类特殊的叠氮化合物, 不仅具有叠氮的化学特性, 而且因茮基的存在使得其应用进一步拓展. 例如可以被 看成是氮烯的替代物, 在有机反应中转化为亲核性的阴 离子或者是亲电性的阳离子等, 进而实现多种类型的化 学反应 ${ }^{[3]}$.
烯烃及其衍生物化学的研究是有机化学的一个重 要研究领域, 与 $\mathrm{sp}^{3}$ 杂化烷烃和 $\mathrm{sp}$ 杂化炔烃相比, $\mathrm{sp}^{2}$ 杂 化烯烃具有中间的氧化态，具有与过渡金属和稀土金属 很强的配位能力, 因此会发生一系列的氧化还原反应或 偶联反应等. 近年来, 随着过渡金属和稀土金属催化反 应的兴起，催化烯烃在温和条件下的新反应引起了合成 化学家的广泛关注. 虽然已经报道了许多重要的结果, 但利用烯烃开发出更新颖的有机合成方法仍然是合成 化学家的一大挑战.

近年来，随着有机含氮化合物的合成及应用范围的 不断扩展，有机化学家对有机叠氮化合物的研究兴趣十

\footnotetext{
* Corresponding authors. E-mail: lzjx0915@163.com; tanmx00@163.com Received March 1, 2019; revised March 30, 2019; published online April 16, 2019. Project supported by the National Natural Science Foundation of China (No. 21761033), the Natural Science Foundation of Guangxi (Nos. 2017GXNSFBA198211, 2018GXNSFAA294064), and the Yulin Normal University Research (Nos. 2018YJKY36, 201810606010). 国家自然科学基金(No. 21761033)、广西自然科学基金(Nos. 2017GXNSFBA198211, 2018GXNSFAA294064)以及玉林师范学院科研(Nos. 2018YJKY36, 201810606010)项目资助.

$\dagger$ 共同第一作者(These authors contributed equally to this work).
} 
分浓厚 ${ }^{[4]}$, 以芳甲基叠氮化合物为代表的叠氮化合物与 烯烃的有机反应更是受到研究者的广泛关注. 对 2014 年至 2018 年以来报道的芳甲基叠氮化合物作为 $\mathrm{N}$ 源与 烯烃及其衍生物的有机反应进行了归纳, 分类介绍了芳 甲基叠氮化合物与烯烃及其衍生物在不同反应中的应 用及相关的反应机理.

\section{1 芳甲基叠氮化合物与烯烃反应}

将结构简单的非极性烯烃作为构建具有复杂结构 分子合成模块, 是有机合成化学和有机合成方法学经常 使用的一种策略. 与其他活性烯烃相比, 苯乙烯和未活 化芳烃是最常见的低反应活性烯烃. 如何使它们参与化 学反应, 将比活性烯烃的研究更具挑战性.

\section{1 与末端烯烃的反应}

2015 年, 潘英明课题组 ${ }^{[5]}$ 报道了利用氯化亚铜作为 催化剂, 在甲苯的溶剂中实现了芳甲基叠氮与末端烯烃 的环化反应，合成了一系列 2,5-二取代恶唑环衍生物 (Eq. 1). 该小组最开始利用苯甲基叠氮与苯乙烯作为模 板底物在不同条件下进行反应, 苯乙烯与 1.2 equiv.的苯 甲基叠氮在 $10 \mathrm{~mol} \%$ 的氯化亚铜 $(\mathrm{CuCl})$ 催化作用下, 可 以得到较高收率的 2,5-二苯基噁唑产物, 产率达到了 $82 \%$. 该方法反应条件温和，当反应体系在氮气的环境 中时, 该反应不能发生, 说明空气中的 $\mathrm{O}_{2}$ 参与了反应并 起着关键性的作用. 该小组对此反应的机理做了比较详 细的研究, 推断该反应首先经历了 1,3 -偶极环加成反应 得到五元环中间体, 因其不稳定会开环并发生 1,2-H 迁 移，随之放出一分子的 $\mathrm{N}_{2}$ 得到烯胺中间体，质谱数据也 证实了此中间体的存在. 然后在氯化亚铜及氧气的双重 催化作用下最终得到目标产物(Scheme 1).

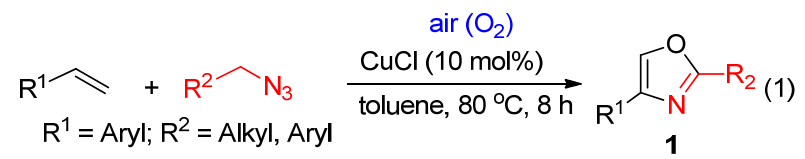

\section{2 与非末端烯烃的反应}

Ruchirawat 小组 ${ }^{[6]}$ 利用三氟乙酸作为催化剂, 在室 温条件下实现了对芳甲基叠氮与不同烯类化合物的 [4+2]环化反应，合成了一系列具有高度立体选择性四 氢哇啉类化合物 (Scheme 2). 当反应底物为含有不饱和 键的炔烃时, 得到的是取代喹啉类化合物. 首先在强酸 的催化作用下, 芳甲基叠氮化合物形成了 $N$-芳基亚胺 正离子中间体, 然后在富电子的烯烃/炔烃存在下与芳 甲基叠氮发生环化反应. 该反应原料底物范围较广, 无 论是烯烃类还是炔烃类底物都可以参与反应, 且不受取 代基的影响, 为合成取代喹啉/四氢喹啉类化合物提供

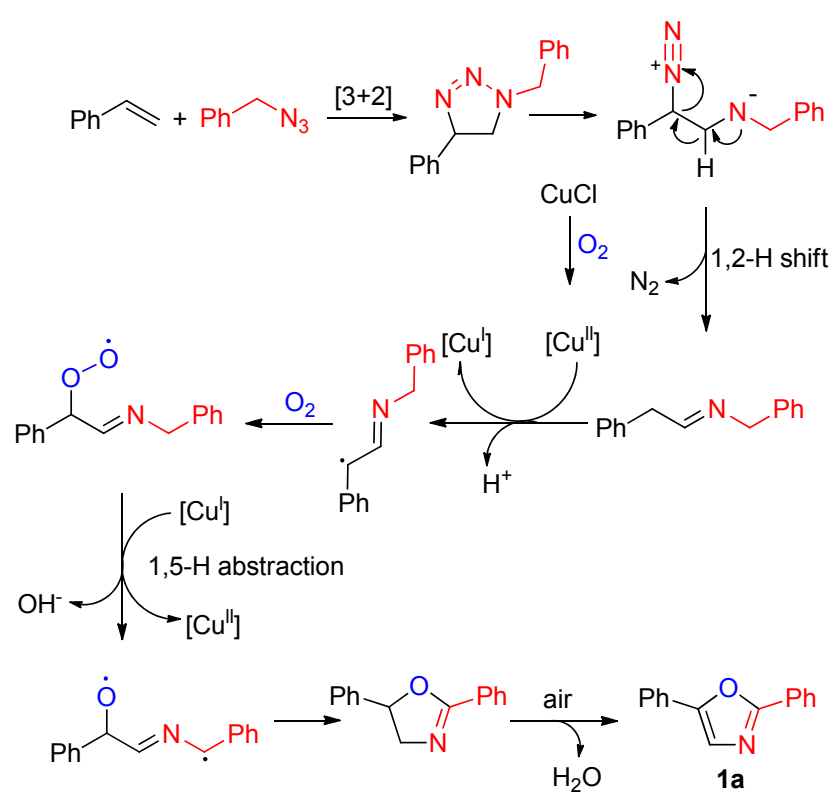

图式 1 可能的反应机理

Scheme 1 Possible reaction mechanism

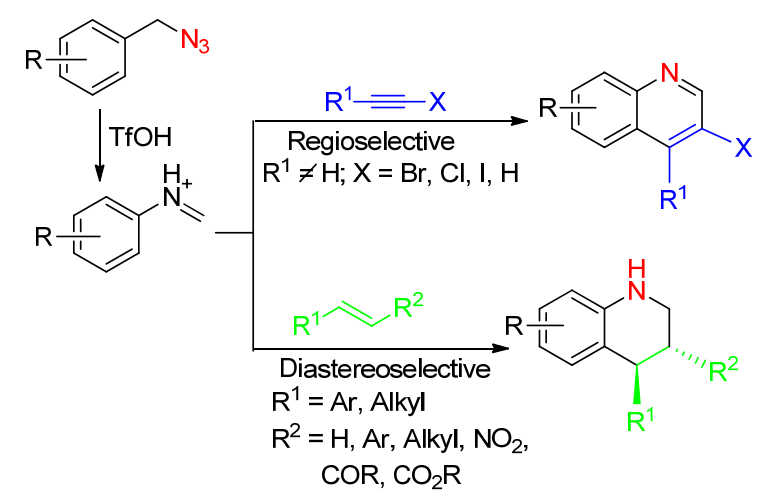

图式 2 芳甲基叠氮化合物与烯烃/炔烃的 [4+2]环加成反应 Scheme 2 [4+2] cycloaddition reaction of aromatic methyl azides with alkenes/alkynes

\section{了一条较好的合成途径.}

\section{3 与共轭二烯烃的反应}

2015 年, 王恒山小组 ${ }^{[7]}$ 使用 $\operatorname{Sm}(\mathrm{OTf})_{3}$ 作为催化剂, 在氧气存在的条件下实现了对芳甲基叠氮与二芳基乙 烯类化合物的环化反应，合成了一系列多取代咪唑类化 合物. 当反应底物的烯烃为共轭二烯烃时，得到的是多 取代吡啶类化合物. 反应以甲苯作为溶剂, 仅需要 5 mol\%催化剂量就可以高效得到目标产物(Scheme 3). 对 于反应机理, 该小组做了比较详细的探究. 在实验的过 程中不仅通过产物的单晶结构进行了佐证, 还加入了理 论计算来证实反应经历了 $1,2-\mathrm{H}$ 迁移/1,2-芳基迁移/环化 /氧化等过程. 作为反应底物的二芳基乙烯类适用范围 较广，无论是末端芳基烯烃还是非末端芳基烯烃，都可 以较好地参与反应. 


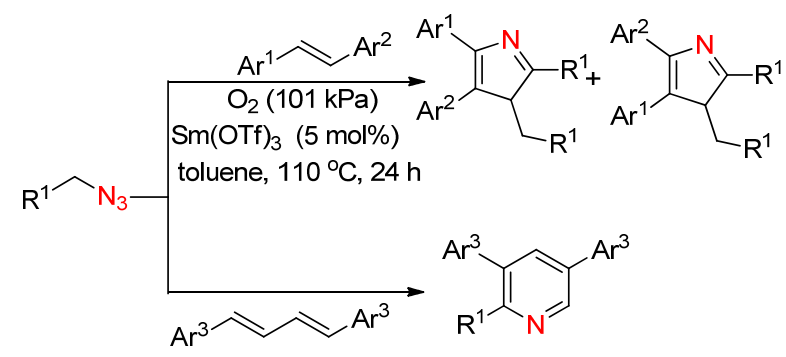

图式 3 芳甲基叠氮化合物与烯烃的 $[2+2+1]$ 环加成反应 Scheme $3[2+2+1]$ cycloaddition reaction of aromatic methyl azides with alkenes

\section{2 芳甲基叠氮化合物与硝基烯烃/烯腈反应}

硝基烯烃/烯腈类化合物因其结构上具有较强的吸 电子基团与烯烃相连, 极大地降低了烯烃双键上的电子 云密度, 从而降低了烯烃的反应活性. 相比末端烯烃/共 轭烯烃而言, 其参与有机反应的报道却相对较少. 如何 实现硝基烯烃/烯腈类化合物与芳甲基叠氮的反应也引 起了有机化学家的广泛关注.

\section{1 与硝基烯烃的反应}

潘英明小组在这一方面做了比较详细的研究. 2014 年, 该小组 ${ }^{[8]}$ 报道利用 $\mathrm{Ce}(\mathrm{OTf})_{3}$ 催化芳甲基叠氮与硝基 苯乙烯的 $[3+2]$ 环加成反应, 高效地合成了 1,5 -二取代 三唑类化合物. 该方法突破了以往传统合成三唑类化合 物的产率低 ${ }^{[9]}$ 、反应时间长 ${ }^{[10]}$ 、底物适用范围窄 ${ }^{[1]}$ 等缺 陷，实现了一种利用芳甲基叠氮构建三唑化合物的新方 法. 该反应催化体系简单、无需任何酸碱催化剂的参与; 无论是芳甲基叠氮还是芳基叠氮都能够有较好的反应, 产率较高(Scheme 4). 对于反应机理, 该小组提出了环 加成一消除过程, 芳甲基叠氮化合物在催化剂的作用下 被活化, 随之与硝基苯乙烯发生 $[3+2]$ 环加成得到五元 环的中间体, 在加热的情况下失去一分子的亚硝酸得到 目标产物.

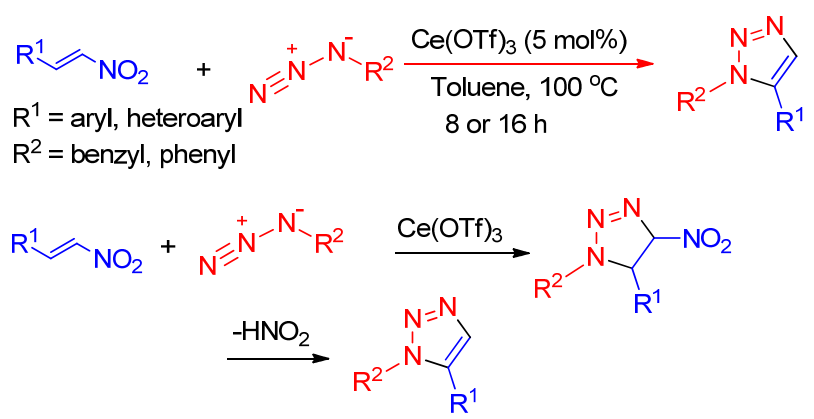

图式 4 可能的反应机理

Scheme 4 Possible reaction mechanism

\section{2 与烯腈的反应}

2015 年, 王恒山小组 ${ }^{[12]}$ 报道了 $\operatorname{Sc}(\mathrm{OTf})_{3}$ 催化芳甲
基叠氮与 $\alpha, \beta$-不饱和烯腈的反应，以甲苯作为反应介质， $110{ }^{\circ} \mathrm{C}$ 下反应 $12 \mathrm{~h}$ 合成具有高立体选择性的 $(\mathrm{Z})-\beta$-烯胺 腈类化合物. 反应不受底物影响, 后处理简单, 产率较 高, 环境友好, 为烯胺腈类化合物的合成提供了一条便 利的途径. 该小组对于反应机理也做了比较详细的研 究, 运用同位素 D 标记法, 揭示该反应经历了 1,3-偶极 环加成/1,2-芳基迁移/1,3-H 转移过程, 并给出了合理的 反应机理(Scheme 5).

$\mathrm{R}^{1} \curvearrowright \mathrm{CN}+\mathrm{R}^{2} \mathrm{~N}_{3} \frac{\mathrm{Sc}(\mathrm{OTf})_{3}(5 \mathrm{~mol} \%)}{\text { toluene, } 110^{\circ} \mathrm{C}, 12 \mathrm{~h}} \overbrace{\mathrm{R}^{1}}^{\mathrm{R}^{2}} \overbrace{\mathrm{N}^{-}}^{-\mathrm{H}}$

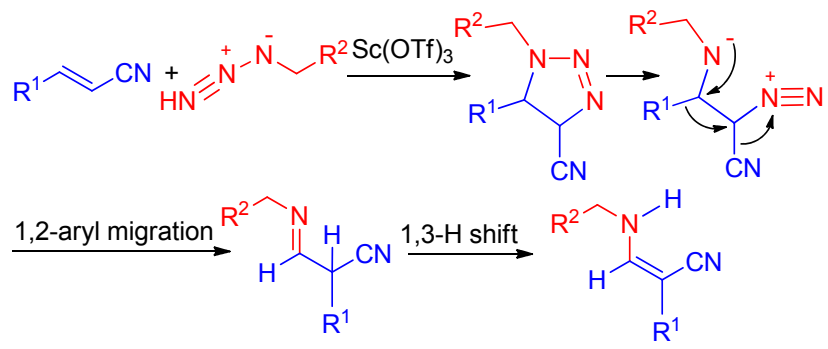

图式 5 可能的反应机理

Scheme 5 Possible reaction mechanism

\section{3 芳甲基叠氮化合物与 $\alpha, \beta$-不饱和羰基化合物 反应}

$\alpha, \beta$-不饱和羰基化合物具有与 $\alpha, \beta$-不饱和烯腈、硝 基苯乙烯等相似的结构, 烯烃双键与吸电子效应的羰基 基团共轭, 不仅降低了烯烃双键上的电子云密度, 而且 因为共轭效应影响极大地降低了烯烃的反应活性. 自 Donald 等 ${ }^{[13]}$ 报道了 $\alpha, \beta$-不饱和羰基化合物与叠氮在酸 性条件下合成烯胺酮以来, 利用叠氮化合物与 $\alpha, \beta$-不饱 和羰基化合物的反应合成内酰胺 ${ }^{[14]}$ 、烯胺酩 ${ }^{[15]}$ 、三元 $\mathrm{N}$ 杂环酮 ${ }^{[16]}$ 以及取代喹啉 ${ }^{[17]}$ 等的反应也逐渐兴起.

2014 年, 潘英明小组 ${ }^{[18]}$ 利用查尔酮与芳甲基叠氮 作为反应底物, 在 $\mathrm{Ce}(\mathrm{OTf})_{3}$ 催化作用下, 通过改变不同 的反应溶剂可以高选择性地合成 $\beta$-芳基烯胺酮或者 $1,4,5$-三取代三唑类化合物。该小组最开始利用苯甲基 叠氮与苯基查尔酮作为模板底物, 在不同条件下进行反 应，苯基查尔酮与 1.2 equiv.的苯甲基叠氮在 $5 \mathrm{~mol} \%$ 的 $\mathrm{Ce}(\mathrm{OTf})_{3}$ 催化作用下反应 $5 \mathrm{~h}$, 以 $N, N$-二甲基甲酰胺作 为溶剂时, 可以得到较高收率的 $\beta$-芳基烯胺酮产物, 产 率达到了 $82 \%$. 当换成甲苯作为溶剂时, 得到的产物却 是 1,4,5-三取代三唑类化合物, 产率达到了 $85 \%$. 目标 产物均通过了单晶结构得到证实. 对此反应的机理做了 大致的推断，首先在催化剂的作用下查尔酮与芳甲基叠 氮化合物经历 1,3-偶极环加成得到五元环中间体, 当甲 
苯作为反应介质时, 该中间体会在空气中失去水得到 1,4,5-三取代三唑类化合物. 改变反应介质为 $N, N$-二甲 基甲酰胺, 得到的五元环中间体会发生开环, 随之发生 1,2-H 迁移/1,3-H 迁移等历程, 得到 $\beta$-芳基烯胺酮产物 (Scheme 6).

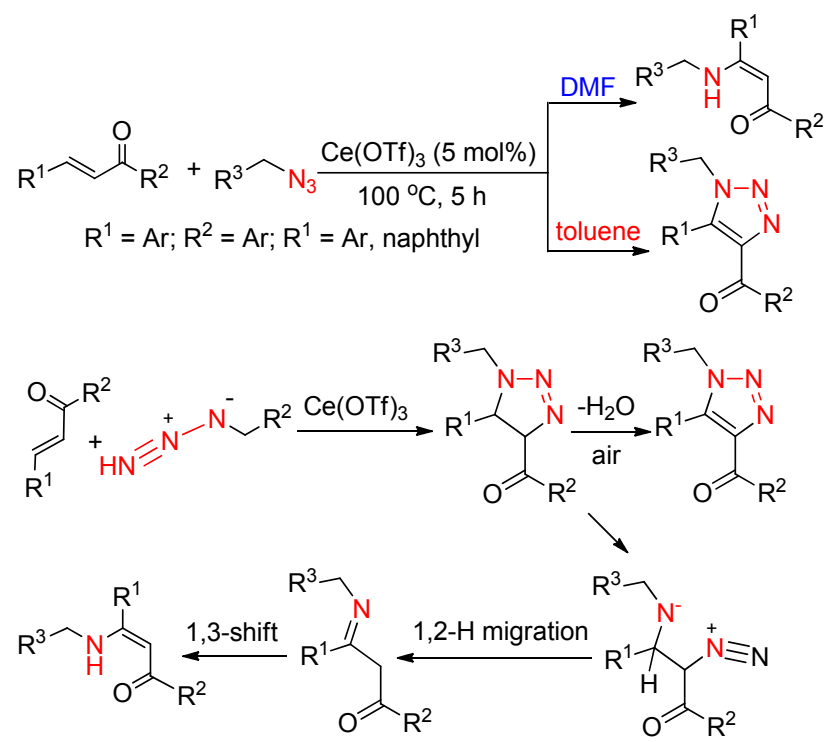

图式 6 可能的反应机理

Scheme 6 Possible reaction mechanism

2015 年, Elangovan 小组 ${ }^{[19]}$ 利用纳米氧化铜催化氧 化芳基叠氮与不饱和烯烃，通过 $[3+2]$ 环加成反应高效 合成 1,2,3-三唑类化合物. 该小组以芳甲基叠氮化合物 与 3 equiv. 的甲基乙烯基酮作为模板反应底物, 首先尝 试了在不同的铜催化剂作用下不同的溶剂介质中的反 应. 发现在 $20 \mathrm{~mol} \%$ 催化量的纳米氧化铜, 在水作为反 应溶剂时能够在室温下高效得到 1,4-二取代三唑, 收率 达到了 $99 \%$. 在对反应进行底物拓展时发现, 无论底物 是芳甲基叠氮还是芳基叠氮, 都能够与不同的烯烃(活 化烯烃/非活化烯烃)在极性溶剂下高效制备出 1,4,5-三 取代 1,2,3-三唑类化合物. 此外, 该小组发现纳米铜催 化剂可以回收再利用而不丧失其催化活性, 并且该类型 反应不需要任何碱或添加剂(Scheme 7).

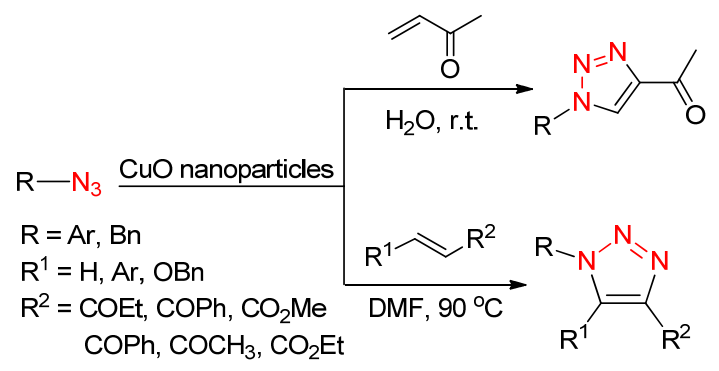

图式 7 芳甲基叠氮化合物与烯烃的 $[3+2]$ 环加成反应 Scheme 7 [3+2] cycloaddition reaction of aromatic methyl azides with alkenes
王䂞小组 ${ }^{[20]}$ 报道了碱催化一氧化查尔酮类化合物与 芳基叠氮的环加成反应。他们以 2 equiv. 的无机碱 $\left(\mathrm{K}_{2} \mathrm{CO}_{3}\right)$ 为碱, 在空气中以 $\mathrm{H}_{2} \mathrm{O} / 1,4$-二氧六环 $(V / V=5 / 1)$ 作为反应介质, $80{ }^{\circ} \mathrm{C}$ 下反应 $11 \mathrm{~h}$ 可以高收率地合成三 取代三唑类化合物(Eq. 2). 该方法无需金属作为催化剂, 合成步骤简单，原子经济性较高，产物收率较好，达到 了 $95 \%$, 符合绿色化学理念. 对于该反应机理, 该小组 提出了 1,3-偶极环加成/1,3-H 迁移/氧化等反应过程 (Scheme 8).
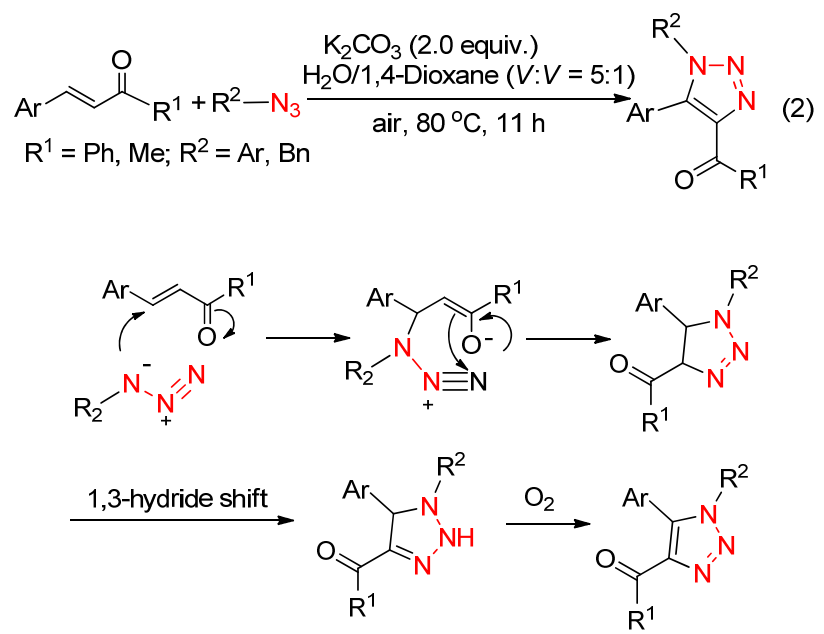

图式 8 可能的反应机理

Scheme 8 Possible reaction mechanism

2016 年, Elangovan 小组 ${ }^{[21]}$ 在芳基叠氮与烯烃类化 合物的反应中有新发现. 该小组以四甲基吡啶氧化物 (TEMPO)作为氧化剂, 在无任何金属催化剂参与的情况 下, 与氧气共催化氧化叠氮与缺电子烯烃的 [3+2]环加 成反应构建 1,4-二取代以及 $1,4,5$-三取代 1,2,3-三唑类化 合物(Eq. 3). 该反应以水作为反应介质，反应条件温和， 底物的适用范围都较广, 无论是开链还是环状的缺电子 烯烃，都能够高产率地得到一系列的目标产物. 该小组 通过实验条件的探索，发现氧气在反应中取到了至关重 要的作用, 在反应过程中多次参与了氧化循环历程, 促 使该反应的进行(Scheme 9).

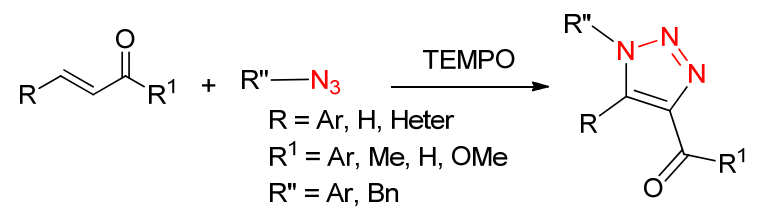

受到 Elangovan 小组的研究启发, 潘英明小组 ${ }^{[22]}$ 继 续在叠氮与不饱和烯烃类化合物的反应中进行新的研 究. 该小组直接选用邻取代茮溴基的 $\alpha, \beta$-不饱和烯烃与 叠氮化钠作为反应底物, 在无任何催化剂及添加剂的参 与下，以 $\mathrm{DMF}$ 作为反应溶剂实现了 $1,2,3$-三唑类化合物 


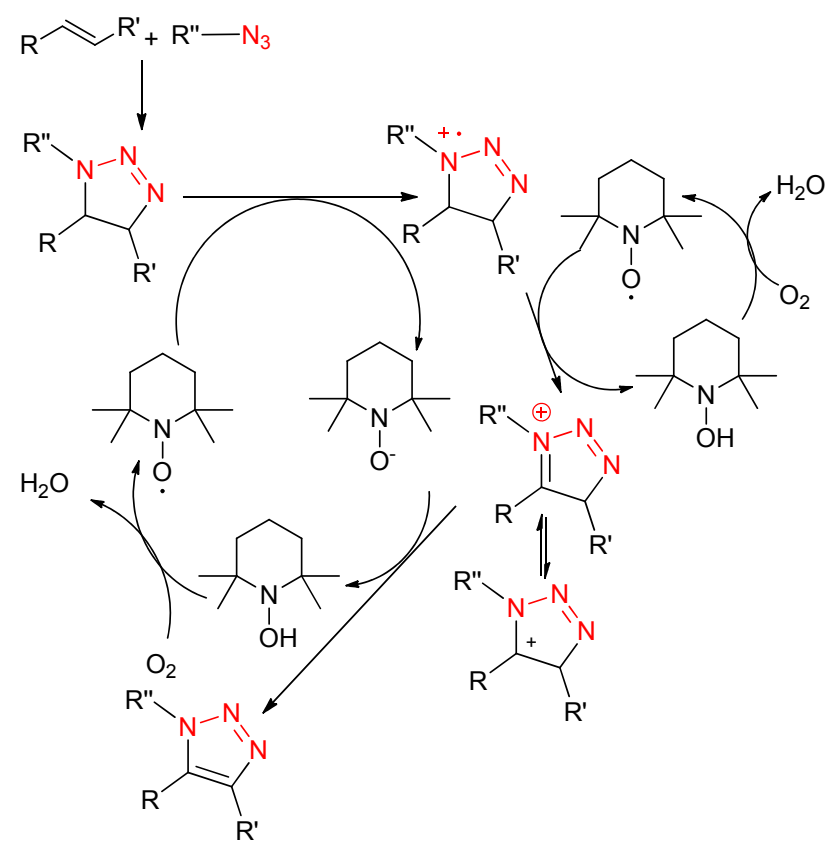

图式 9 可能的反应机理

Scheme 9 Possible reaction mechanism

以及异喹啉类化合物的选择性合成. 在室温下反应时, 可以得到较好收率的 1,2,3-三唑类化合物; 通过改变反 应温度及增加氮气保护, 可以得到高产率的异喹啉类化 合物. 这一发现为 $1,2,3$-三唑类化合物以及异喹啉类化 合物的合成提供了一条简洁高效的路线(Scheme 10).

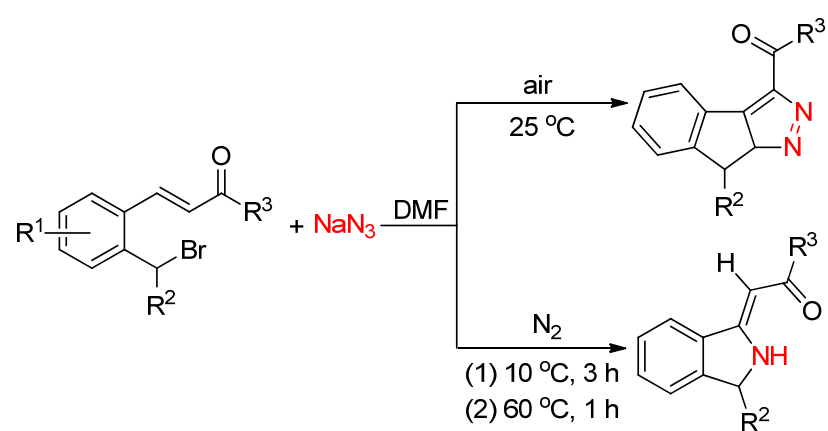

图式 10 1,2,3-三唑类化合物以及异喹啉类化合物的合成

Scheme 10 Synthesis of fused 1,2,3-triazole and isoindoline derivatives

乙酰乙酸乙酯类化合物因其酮式与烯醇式互变异 构性质，也被应用于与叠氮化合物的研究中. 2014 年, 潘英明小组 ${ }^{[23]}$ 利用一锅法分步合成策略, 以苠卤、叠氮 化钠以及 1,3-二酮类化合物为反应原料, 在叔丁醇钾的 催化作用下得到了烯胺酮类化合物(Eq. 4). 该反应以 $\mathrm{DMF}$ 作为反应介质, 反应条件温和, 底物的适用范围都 较广, 无论是苄氯还是芐澳都能够与不同的 1,3 -二酮类 化合物反应得到一系列的烯胺酮类化合物, 目标产物的 构型也通过单晶得到证实. 该小组对其反应机理做出了 推测(Scheme 11). 茮卤与叠氮化钠首先得到芳甲基叠氮
化合物，在强碱的作用下形成具有较强亲核能力的中间 体，再与乙酰乙酸乙酯发生加成反应，随后经历多次的 质子转移/去氮等历程达到目标产物。

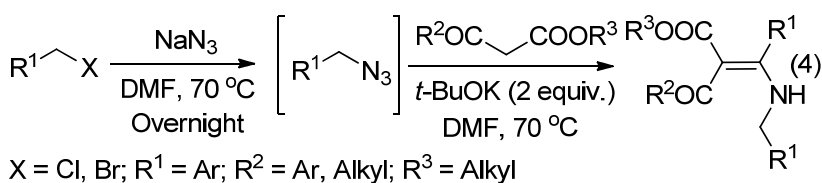

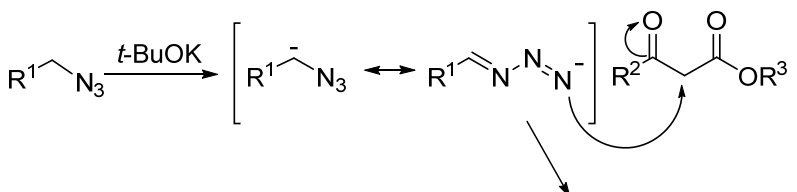

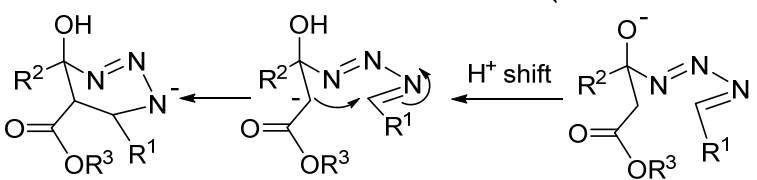
$\mathrm{H}^{+}$shift

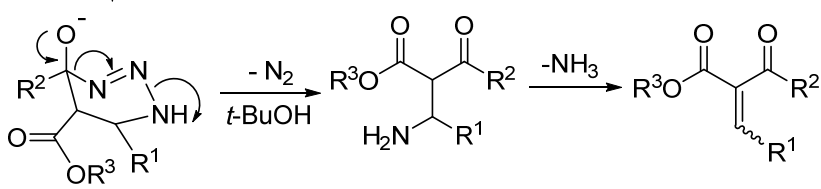

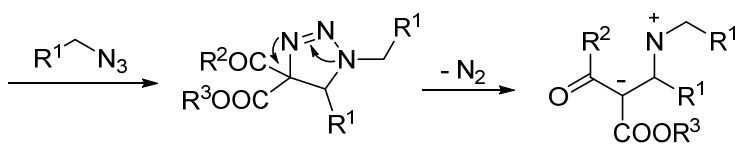

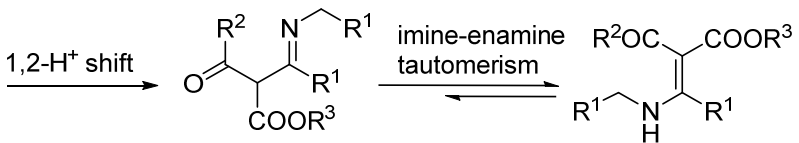

图式 11 可能的反应机理

Scheme 11 Possible reaction mechanism

\section{4 展望}

芳甲基叠氮化合物能够在有机反应中较好地引入 $\mathrm{N}$ 源，与烯烃及其衍生物反应可以合成许多具有药物活 性的含氮有机物, 具有重大的研究意义. 芳甲基叠氮化 合物具有稳定、合成简单等特点，在有机合成化学领域 有广泛的应用. 但目前研究芳甲基叠氮化合物与烯烃及 其衍生物反应大多采用金属催化剂，如何通过改变反应 条件实现酸、碱以及有机小分子催化反应将是此类有机 反应的主要研究方向.

\section{References}

[1] Grecianl, S.; Aubé, J. Organic Azides: Syntheses and Applications, Vol. 7, Eds.: Bräse, S.; Banert, K., John Wiley \& Sons, Ltd, Chichester, UK, 2010, pp. 191 310.

[2] (a) Bräse, S.; Gil, C.; Knepper, K.; Zimmermann, V. Angew. Chem., Int. Ed. 2005, 44, 5188.

(b) Lee, J. H.; Gupta, S.; Jeong, W.; Rhee, Y. H.; Park, J. Angew. Chem., Int. Ed. 2012, 51, 10851.

(c) Han, J.; Jeon, M.; Pak, H. K.; Rhee, Y. H.; Park, J. Adv. Synth. 
Catal. 2014, 356, 2769.

(d) Gupta, S.; Han, J.; Kim, Y.; Lee, S. W.; Rhee, Y. H.; Park. J. J. Org. Chem. 2014, 79, 9094.

(e) Chou, H.-H.; Raines, R. T. J. Am. Chem. Soc. 2013, 135, 14936. (f) Zhang, X.-X.; Sun, X.-P.; Zhang, H.-F.; Cui, X.-L.; Ma, M.-T. Chin. J. Org. Chem. 2015, 35, 1469 (in Chinese).

(张小祥, 孙小萍, 张海飞, 崔杏丽, 马猛涛, 有机化学, 2015, 35,1469 .)

(g) Zhang, W.-S.; Xu, W.-J.; Kuang, C.-X. Chin. J. Org. Chem. 2015, 35, 2059 (in Chinese).

(张文生，许文静，匡春香，有机化学, 2015, 35, 2059.)

[3] (a) Song, Z.-Q.; Zhao, Y.-M.; Zhai, H.-B. Org. Lett. 2011, 13, 6331 (b) Lamani, M.; Devadig, P.; Prabhu, K. R. Org. Biomol. Chem. 2012, 10, 2753.

(c) Tummatorn, J.; Thongsornkleeb, C.; Ruchirawat, S.; Gettongsong, T. Org. Biomol. Chem. 2013, 11, 1463.

[4] Shin, K.; Kim, H.; Chang, S. Acc. Chem. Res. 2015, 48, 1040.

[5] Li, J.-L.; Wang, Y.-C.; Li, W.-Z.; Wang, H.-S.; Mo, D.-L.; Pan, Y.-M. Chem. Commun. 2015, 51, 17772.

[6] Tummatorn, J.; Poonsilp, P.; Nimnual, P.; Janprasit, J.; Thongsornkleeb, C.; Ruchirawat, S. J. Org. Chem. 2015, 80, 4516.

[7] Wang, Y.-C.; Li, J.-L.; He, Y.; Xie, Y.-Y.; Wang, H.-S.; Pan, Y.-M. Adv. Synth. Catal. 2015, 357, 3229.

[8] Wang, Y.-C.; Xie, Y.-Y.; Qu, H.-E.; Wang, H.-S.; Pan, Y.-M.; Huang, F. -P. J. Org. Chem. 2014, 79, 4463.

[9] Zefirov, N. S.; Chapovskaya, N. K.; Kolesnikov, V. V. Chem. Commun. 1971, 1001.
[10] Piet, J. C.; Le H. G.; Cailleux, P.; Benhaoua, H.; Carrie, R. Bull. Soc. Chim. Belg. 1996, 105, 33.

[11] Amantini, D.; Fringuelli, F.; Piermatti, O.; Pizzo, F.; Zunino, E.; Vaccaro, L. J. Org. Chem. 2005, 70, 6526.

[12] Wang, Y.-C.; Xie, Y.-Y.; Tan, X.-C.; Wang, H.-S.; Pan, Y.-M. Org Biomol. Chem. 2015, 13, 513.

[13] Donald, A. S. R.; Marks, R. E. J. Chem. Soc. C 1967, 1188.

[14] Casey, M.; Donnelly, J. A.; Ryan, J. C.; Ushioda, S. ARKIVOC 2003, 7,310.

[15] (a) Reddy, D. S.; Judd, W. R.; Aubé, J. Org. Lett. 2003, 5, 3899. (b) Silvio, C.; Amenson, T. G. Tetrahedron Lett. 2012, 53, 6710

[16] Mahoney, J. M.; Smith, C. R.; Johnston, J. N. J. Am. Chem. Soc. 2005, $127,1354$.

[17] Jumreang, T.; Charnsak, T.; Somsak, R.; Tanita, G. Org. Biomol. Chem. 2013, 11, 1463.

[18] Xie, Y.-Y.; Wang,Y.-C.; Qu, H.-E.; Tan, X.-C.; Wang, H.-S.; Pan, Y.-M. Adv. Synth. Catal. 2014, 356, 3347.

[19] Gangaprasad, D.; Paul Raj, J.; Kiranmye, T.; Sagubar Sadik, S.; Elangovan, J. RSC Adv. 2015, 5, 63473.

[20] Yang, W.-C.; Miao, T.; Li, P.-H.; Wang, L. RSC Adv. 2015, 5, 95833.

[21] Gangaprasad, D.; Paul Raj, J.; Kiranmye, T.; Karthikeyan, K.; Elangovan, J. Eur. J. Org. Chem. 2016, 5642.

[22] Xie, Y.-Y.; Wang, Y.-C.; He, Y.; Hu, D.-C.; Wang, H.-S.; Pan, Y.-M. Green Chem. 2017, 19, 656.

[23] Yan, Z.-M.; Wu, N.; Liang, D.; Wang, H.-S.; Pan, Y.-M. Org. Lett. 2014, 16, 4048. 